\title{
Síndrome de Burnout em fisioterapeutas de um hospital público de alta complexidade da cidade do Recife, Pernambuco
}

\section{Burnout syndrome in physiotherapists at a high complexity hospital in the city of Recife, State of Pernambuco - Brazil}

Ericky Rafael Santos ${ }^{1}$, Luana Valeriano Neri² ${ }^{2}$ Elaine Lima Silva Wanderley ${ }^{1}$

\section{RESUMO}

Por serem expostos às inadequadas condições de trabalho, os Fisioterapeutas podem desenvolver a síndrome de Burnout (SB), que é uma resposta ao estresse crônico associado ao ambiente ocupacional. Objetivo: Investigar a frequência da SB em Fisioterapeutas de um hospital público, verificando associações com variáveis demográficas e laborais. Métodos: Este estudo foi desenvolvido no Hospital da Restauração Governador Paulo Guerra, em 2015. Foram incluídos Fisioterapeutas atuantes no hospital e excluídos aqueles com história de depressão ou outros transtornos emocionais. O questionário autoaplicável Maslach Burnout Inventory foi utilizado para investigar a SB. Estatística descritiva foi utilizada para caracterizar a amostra e determinar a frequência da SB, a regressão multivariada foi utilizada para verificar relações entre a SB e as variáveis demográficas e laborais $(p<0,05)$. Resultados: Participaram deste estudo 48 fisioterapeutas. A maioria atuava nas unidades de terapia intensiva $(56,3 \%(N=27))$. A presença da SB foi identificada em $54,2 \%(N=26)$ dos participantes. Foram observadas correlações entre o número de atendimentos diários e a exaustão emocional $(r=0,41)$ e com a realização pessoal $(r=-0,30)$, e da idade com a despersonalização $(r=-0,11)$. Conclusão: A SB foi verificada em mais da metade dos Fisioterapeutas. Correlações positivas foram observadas entre o número de atendimentos diários e a exaustão emocional e correlação negativa entre a idade com despersonalização e número de atendimentos diários com a realização pessoal.

Palavras-chave: Saúde do Trabalhador, Esgotamento Profissional, Fisioterapeutas

${ }^{1}$ Instituto Pernambucano de Ensino Superior IPESU.

${ }^{2}$ Faculdade do Recife.

\section{Correspondência}

Ericky Rafael Santos

E-mail: ericky_rafael@hotmail.com

Recebido: 07 Junho 2018.

Aceito: 10 Outubro 2018.

\section{Como citar}

Santos ER, Nari LV, Wanderley ES. Síndrome de Burnout em fisioterapeutas de um hospital público de alta complexidade da cidade do Recife, Pernambuco. Acta Fisiatr. 2018;25(1):31-35.

\begin{abstract}
As they are exposed to inadequate working conditions, physiotherapists may acquire Burnout Syndrome (BS), a body response to chronic stress which is associated to the work environment. Objective: The objective is to investigate the occurrence of BS among physiotherapists at a state hospital and correlate the disease with demographic and labor variables. Methods: This study was conducted at the Paulo Guerra Governor Restoration Hospital, in 2015. This study included physiotherapists who were actively working at the hospital, and those with a history of depression or other emotional disorders were excluded. The self-applicable Maslach Burnout Inventory was used. Descriptive statistics was then used to characterize the sample and establish the frequency of BS; multiple regression analysis was used to establish relations between BS and the demographic and labor variables. Results: 48 physiotherapists were included in this study. Most of these professionals were working in ICUs $(56.3 \%, \mathrm{~N}=27)$. The presence of BS was identified in $54.2 \%$ of the participants $(\mathrm{N}=26)$. Correlations were found between the number of daily physical therapy sessions and emotional exhaustion $(r=0.41)$, and with personal accomplishment $(r=-0.30)$, as well as between age and depersonalization $(r=0.11)$. Conclusion: BS was observed in over half the physiotherapists. Positive correlations were observed between the number of cases dealt with daily and emotional exhaustion, whereas negative correlation between age with depersonalization and the number of daily physical therapy sessions with personal accomplishment were found.
\end{abstract}

Keywords: Occupational Health, Burnout, Professional, Physical Therapists 


\section{INTRODUÇÃO}

A síndrome de Burnout (SB) ou síndrome do esgotamento profissional é descrita como uma resposta à cronificação do estresse vivenciado no ambiente de trabalho, em decorrência de sentimentos e condutas negativas, que geram prejuízos no contexto profissional, pessoal, familiar e social. ${ }^{1,2}$ A SB têm se apresentado como um problema de saúde pública em vários países, incluindo o Brasil. ${ }^{3}$ Dados do Ministério da Previdência Social indicaram 592 beneficiários devido ao esgotamento (CID Z73.0), entre os anos de 2010 e $2014 .^{4}$

Maslach e Jackson ${ }^{5}$ descreveram a SB através de três dimensões: Exaustão Emocional (EE), Despersonalização (DP) e Realização Profissional/Pessoal (RP). ${ }^{6}$ A EE é caracterizada pela ausência ou déficit de energia associada à sensação de que todos os recursos emocionais e físicos foram esgotados. Já a DP apresenta-se como a perda da sensibilidade emocional associada a reações negativas e impiedosas direcionadas àqueles para os quais presta serviços. Por fim, a RP caracteriza-se por autoavaliação negativa por parte do profissional sobre o seu desempenho do ambiente de trabalho e pessoal. ${ }^{5}$

A etiologia da SB é desconhecida, porém estudos revelam que sobrecarga de trabalho, falta de incentivos, recompensas injustas, relações de trabalho interpessoal inadequadas e o conflito entre o profissional e o ambiente ocupacional são desencadeantes da SB. ${ }^{7,8}$ Estudos têm demonstrado que profissionais de diferentes áreas relacionadas à saúde apresentaram o quadro da SB, devido às características deste tipo de trabalho. ${ }^{9}$

Fazendo parte deste grupo de profissionais, o fisioterapeuta está exposto a cargas horárias excessivas, baixa remuneração e reconhecimento profissional, inadequadas condições de trabalho, a riscos físicos e químicos, além da condição de tensão decorrente do contínuo elo interpessoal com os pacientes. ${ }^{10}$

Todas essas condições se agravam no âmbito hospitalar, onde é constante o contato com a dor, sofrimento e o óbito. ${ }^{8}$ Segundo o estudo de Al-Imam e Al-Sobayel ${ }^{8}$ o desenvolvimento da SB em Fisioterapeutas não afeta apenas o profissional, pois causa prejuízos diretos na execução das técnicas fisioterapêuticas e no relacionamento com o paciente e família.

Com base nessas informações, tornase relevante a realização de estudos sobre a SB em Fisioterapeutas brasileiros, a fim de identificar as causas e características do desenvolvimento da doença nesta população, de forma que possam servir de base para a adoção de políticas públicas, visando à prevenção precoce e uma melhor assistência aos profissionais já acometidos.

\section{OBJETIVO}

Diante disto, o objetivo deste estudo foi caracterizar a presença da SB nos Fisioterapeutas de um hospital público de alta complexidade e verificar possível correlação desta síndrome com variáveis demográficas e laborais.

\section{MÉTODO}

Este estudo observacional foi realizado no Hospital da Restauração Governador Paulo Guerra (HR), entre os meses de Julho e Setembro de 2015 e aprovado pelo Comitê de Ética e Pesquisa do Hospital da Restauração - PE sob o número de CAEE: 36301314.8.0000.5198.

Foram incluídos Fisioterapeutas atuantes no referido hospital, sendo excluídos aqueles que realizaram algum tipo de tratamento para depressão ou outros distúrbios de caráter psicológico antes do vínculo empregatício no referido hospital para evitar a relação dos quadros anteriores com a presença de estresse no trabalho, uma vez que a SB está relacionada exclusivamente às atividades ocupacionais.

Inicialmente os Fisioterapeutas foram recrutados por meio de contato direto no próprio ambiente de trabalho, sendo esclarecidos sobre os objetivos e procedimentos da pesquisa. Todos que aceitaram participar assinaram o Termo de Consentimento Livre e Esclarecido (TCLE). Em seguida, os participantes receberam dois instrumentos autoaplicáveis: o questionário sociodemográfico e ocupacional e o Maslach Burnout Inventory (MBI). Tendo até 7 dias para preenchê-los.

O questionário sociodemográfico e ocupacional foi desenvolvido com a finalidade de caracterizar o perfil dos participantes, sendo composto por itens como: nome, data da entrevista, idade, sexo, número de filhos, estado civil, tempo de serviço no hospital, quantidade de pacientes atendido por plantão, entre outras perguntas relevantes para melhor correlacionar a SB com os dados sociodemográfico e ocupacionais.
O $\mathrm{MBI}$ foi elaborado por Maslach e Jackson $^{5}$ sendo o questionário mais utilizado para caracterizar a SB. ${ }^{11,12}$ Foi traduzido e validado para língua portuguesa por Benevides-Pereira $^{13}$ e possui 22 itens que analisam as três dimensões que representam a SB: EE composta por 9 itens (questões 1, 2, 3, $6,8,13,14,16$ e 20), DP por 5 itens (questões $5,10,11,15$ e 22 ) e RP composta por 8 itens (questões 4, 7, 9, 12, 17, 18, 19 e 21). ${ }^{13}$

As opções de escore para cada item do instrumento original variam de 0 a 65 . Entretanto, no processo de adaptação transcultural para o Brasil as opções de escore foram modificadas, passando a variar dentro de uma escala Likert de 1 a 5: 1 para "nunca", 2 para "raramente", 3 para "algumas vezes", 4 para "frequentemente" e 5 para "sempre". ${ }^{14}$

Cada subescala é pontuada separadamente, de acordo com os critérios: EE (Nível baixo <19, Nível médio entre 1926 , Nível alto $\geq 27$ ), DP (Nível baixo $<6$, Nível médio entre $6-9$, Nível alto $\geq 10$ ) e RP (Nível baixo $\geq 40$, Nível médio entre 34-39, Nível alto $\leq 33) .{ }^{15-19}$ A presença da SB foi determinada, conforme preconizado, nos casos de nível alto de $E E$, ou DP, ou nível baixo de RP (subescala inversa)..$^{17,20,21}$

A estatística descritiva foi utilizada para caracterizar a amostra e determinar a frequência da SB e a análise de regressão múltipla foi utilizada para verificar relações entre o quadro da SB e variáveis demográficas e laborais, considerando-se $\mathrm{p}<0,05$. Foram consideradas como variáveis dependentes a Exaustão emocional, a Despersonalização e a Realização Profissional e como variáveis explicativas a idade, a renda mensal, o tempo de experiência no hospital, o número de atendimentos diários e o número de horas trabalhadas por semana.

Foi realizada uma análise individual para cada variável dependente, sendo utilizado o método ENTER (Entrar) de entrada das variáveis explicativas, o que significa que todas elas foram introduzidas juntas para prever a variável dependente analisada. Além disso, foi utilizado o teste de normalidade de Shapiro Wilk, para a verificação das variáveis dependentes, indicado para amostras $<50$. Para as análises acima descritas foi utilizado o pacote estatístico SPSS for Windows (versão 20.0).

\section{RESULTADOS}

Dos 51 Fisioterapeutas atuantes no hospital, 48 (94,1 \%) aceitaram participar 
deste estudo e responderam os questionários, a maioria atuante nos setores de Unidade de Terapia Intensiva (UTI). Observou-se que a maior parte possuía outros vínculos empregatícios e que a renda mensal média foi maior do que o piso salarial da classe $( \pm \mathrm{R} \$$ $1.200,00)$. Além disso, a maioria trabalhava sob contrato temporário (Tabela 1 ).

Os resultados obtidos através do $\mathrm{MBI}$ demonstraram que $54,2 \%$ ( $N=26$ ) apresentaram a SB. Os valores médios obtidos nas três dimensões foram: $23,56(6,25 \pm D P)$ para $E E$, $8,10(2,49 \pm$ DP) para DP e 31,70 (4,33 \pm DP) para RP. A maioria dos participantes pontuou um nível médio para as subescalas de EE e DP, e um nível alto para a subescala de RP. A dimensão que apresentou um maior percentual de comprometimento (nível alto) foi EE (Figura 1).

A SB foi mais frequente nos Fisioterapeutas que possuíam contratos temporários $(72 \%$ $(\mathrm{N}=18)$ ) do que entre os que possuíam vínculo efetivo $(34,78 \%(N=8))$, naqueles que não gozavam férias regularmente $(75 \%(\mathrm{~N}=12))$ do que entre os que gozavam $(43,75 \%(\mathrm{~N}=14))$ e nos que possuíam outro vínculo $(96,2 \%(\mathrm{~N}=40))$ do que entre os que não possuíam $(3,8 \%$ $(\mathrm{N}=1)$ ). Entre os profissionais que atuavam nas UTI's observou-se maior frequência da SB $(62,96 \%(\mathrm{~N}=17))$ do que entre os das enfermarias $(53,8 \%(N=7))$ ou do ambulatório $(25 \%(N=2))$. A frequência da SB nos homens $(54,5 \%(N=6))$ e nas mulheres $(54,1 \%(N=20))$ foi quase a mesma, assim como entre os que realizavam atividade física $(54,54 \%(\mathrm{~N}=18))$ e os que não realizavam $(53,3 \%(\mathrm{~N}=8))$.

As variáveis dependentes apresentaram distribuição normal. A análise de regressão múltipla demonstrou que a $\mathrm{EE}$ e as variáveis explicativas apresentam correlação moderada $(R=0,48)$, sendo que juntas as variáveis explicativas foram responsáveis por $23 \%$ da variância da EE (R2 ajustado=0,23). Observouse correlação positiva moderada entre a pontuação obtida na dimensão EE e o número de atendimentos diários ( $b=0,40$ com IC $95 \%=$ 0,07 e 0,73 ).

Observou-se também correlação moderada entre as variáveis explicativas e a DP $(R=0,51)$, sendo as variáveis explicativas responsáveis por $26 \%$ da variância de DP $(R 2$ ajustado $=0,26)$. Além disso, verificouse correlações negativas fracas entre DP $(b=$ $-0,11$ com IC $95 \%=-0,20$ e $-0,02$ ).

Por fim, observou-se correlação moderada entre a RP e as variáveis explicativas $(R=0,51)$, sendo estas responsáveis por $26 \%$ da variância da RP (R2 ajustado=0,26). Verificou-se também correlação negativa fraca entre a RP e o número de atendimentos diários ( $b=-0,29$ com IC 95\%=-0,51 e -0,06) (Tabela 2).

Tabela 1. Caracterização dos 48 fisioterapeutas atuantes no Hospital da Restauração Governador Paulo Guerra, Recife-PE, 2015.

\begin{tabular}{|c|c|}
\hline Variável & Resultado \\
\hline \multicolumn{2}{|l|}{ Quadro da SB (N (\%)) } \\
\hline Sim & $26(54,2)$ \\
\hline Não & $22(45,8)$ \\
\hline Idade (média em anos $\pm D P$ ) & $39,90(12,40)$ \\
\hline \multicolumn{2}{|l|}{$\operatorname{Sexo} \%(N)$} \\
\hline Masculino & $22,9(11)$ \\
\hline Feminino & $77,1(37)$ \\
\hline \multicolumn{2}{|l|}{ Estado civil \% (N) } \\
\hline Casado & $35,4(17)$ \\
\hline Divorciado & $12,5(6)$ \\
\hline Solteiro & $39,6(19)$ \\
\hline Viúvo & $4,2(2)$ \\
\hline Outros & $8,4(4)$ \\
\hline \multicolumn{2}{|l|}{ Número de filhos \% (N) } \\
\hline 0 & $45,8(22)$ \\
\hline 1 & $25,0(12)$ \\
\hline 2 & $20,8(10)$ \\
\hline 3 & $8,3(4)$ \\
\hline \multicolumn{2}{|l|}{ Setor em que atua \% (N) } \\
\hline Ambulatórios & $16,7(8)$ \\
\hline Enfermarias & $27,1(13)$ \\
\hline UTIs & $56,3(27)$ \\
\hline \multicolumn{2}{|l|}{ Vínculo empregatício \% (N) } \\
\hline Efetivos & $47,9(23)$ \\
\hline Contratados & $52,1(25)$ \\
\hline \multicolumn{2}{|l|}{ Possui outros vínculos \% (N) } \\
\hline $\operatorname{sim}$ & $87.5(42)$ \\
\hline Não & $12,5(6)$ \\
\hline \multicolumn{2}{|l|}{ Relato de prática de atividade Física \% (N) } \\
\hline Sim & $69,7(33)$ \\
\hline Não & $31,3(15)$ \\
\hline \multicolumn{2}{|l|}{ Relata gozar férias regularmente \% (N) } \\
\hline Sim & $66,7(32)$ \\
\hline Não & $33,3(16)$ \\
\hline \multicolumn{2}{|l|}{ Realiza psicoterapia \% (N) } \\
\hline Sim & $8,3(4)$ \\
\hline Não & $91,7(44)$ \\
\hline Renda Mensal (média em $\mathrm{R} \$ \pm \mathrm{DP}$ ) & $4543,75(1430,98)$ \\
\hline Tempo de atuação no hospital (média em anos \pm DP) & $10,17(9,97)$ \\
\hline Carga horária semanal no hospital (média em horas \pm DP) & $32,88(14,22)$ \\
\hline Carga horária semanal total (média em horas \pm DP) & $62,96(27,51)$ \\
\hline Atendimentos por dia (média $\pm \mathrm{DP}$ ) & $12,83(5,45)$ \\
\hline
\end{tabular}

UTI= Unidade de Terapia Intensiva / DP= Desvio Padrão

\section{DISCUSSÃO}

Os resultados deste estudo demonstraram que mais da metade dos Fisioterapeutas participantes apresentaram a SB, sendo a
EE a dimensão com maior percentual de comprometimento. A frequência de SB identificada em estudos anteriores que envolveram Fisioterapeutas foi menor do que a encontrada no presente estudo. , $, 8,21,22^{2}$ 


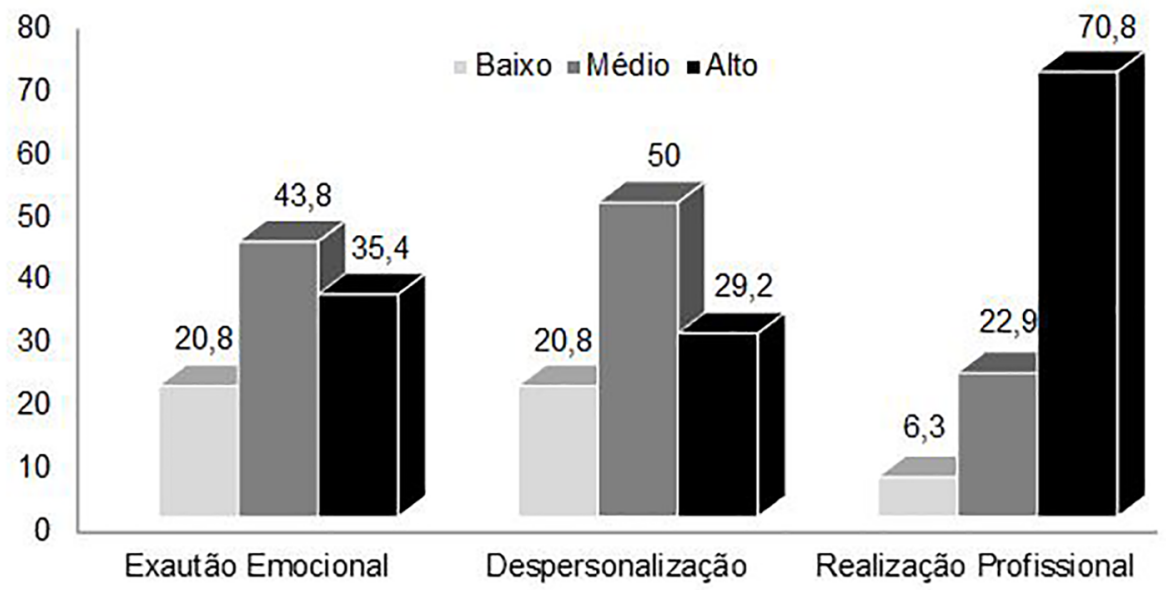

Figura 1. Percentual do nível de pontuação obtida pelos 48 fisioterapeutas nas subescalas: exaustão emocional, despersonalização e realização profissional do questionário Maslach Burnout Inventory (MBI). Hospital da Restauração Governador Paulo Guerra (HR), Recife-PE, 2015.

Tabela 2. Resultado da análise de regressão linear multivariada entre as dimensões exaustão emocional, despersonalização e realização profissional do questionário Maslach Burnout Inventory (MBI) e as variáveis analisadas em 48 fisioterapeutas do Hospital da Restauração Governador Paulo Guerra, Recife-PE, 2015.

\begin{tabular}{lcc}
\hline Exaustão emocional & Despersonalização & Realização profissional \\
\hline$b=-0,87$ & $b=-0,11$ & $b=0,11$ \\
$(p=0,43)$ & $(p=0,01)$ & $(p=0,15)$ \\
$b=-0,00$ & $b=0,00$ & $b=0,33$ \\
$(p=0,24)$ & $(p=0,45)$ & $(p=0,15)$ \\
$b=-0,44$ & $b=0,46$ & $b=0,00$ \\
$(p=0,74)$ & $(p=0.38)$ & $(p=0,97)$ \\
$b=0,40$ & $b=0,06$ & $b=-0,29$ \\
$(p=0,01)$ & $(p=0,32)$ & $(p=0,01)$ \\
$b=0,05$ & $b=0,01$ & $b=-0,00$ \\
$(p=0,20)$ & $(p=0,34)$ & $(p=0.97)$ \\
\hline
\end{tabular}

$\mathrm{b}=$ correlação observada na análise de regressão múltipla (valor de beta).

Nível de significância adotado foi $p<0,05$.

Entretanto, foram observadas diferenças em relação ao tipo de instrumento e os critérios de avaliação utilizados para identificar a SB. Em um destes estudos foi observada uma frequência de 7,5\%, através da utilização do instrumento Maslach Burnout Inventory - Geral (MBIGS), composto por 16 questões divididas em três subescalas, Exaustão, Cinismo e Eficácia Profissional. ${ }^{8}$ Da mesma forma, em um estudo realizado no Brasil, envolvendo 1040 fisioterapeutas, não foram identificados casos de SB, através da utilização do Cuestionario para la Evaluación del Síndrome de Quemarse por el Trabajo (CESQT). ${ }^{22}$

A utilização de diferentes questionários implica em diferentes critérios para a identificação da SB, o que dificulta a comparação destes resultados com os encontrados no presente estudo. ${ }^{8,22}$ Por outro lado, estudos que utilizaram o $\mathrm{MBI}$ encontraram frequências mais baixas do que as observadas no presente estudo. ${ }^{7,21} \mathrm{Em}$ um destes estudos, a frequência foi de 10,87\%, em uma amostra composta por 46 fisioterapeutas, entretanto a SB foi determinada quando houve pontuações altas em EE e DP e baixas em PR. ${ }^{7}$ No presente estudo, optou-se por critério de identificação da SB utilizado em outros estudos envolvendo profissionais de saúde, inclusive Fisioterapeutas. ${ }^{17,20,21}$

Por fim, em um estudo realizado em Cypriot, foi identificada uma frequência de $21,1 \%$ numa amostra de 172 fisioterapeutas. Neste estudo foram utilizados os mesmos critérios diagnósticos do presente estudo, entretanto as condições socioeconômicas que existem em países Europeus são diferentes das observadas no Brasil, o que pode ter influenciado nas frequências. ${ }^{21}$
Os valores médios dos escores obtidos nas três dimensões do $\mathrm{MBI}$ variaram entre diferentes países (Arábia Saudita, Espanha e Japão). Ademais, as médias obtidas nestes estudos demonstraram níveis moderados em EE e DP, e nível alto de RP, corroborando os achados do presente estudo. ${ }^{7,8,23}$ Exceção foi verificada no estudo realizado em Cypriot, onde foram encontrados níveis baixos de EE e $\mathrm{DP}$, mantendo-se um nível alto de RP, porém a amostra incluiu Fisioterapeutas de hospitais públicos e privados. ${ }^{21}$

Observou-se no presente estudo, maior frequência da SB em Fisioterapeutas que possuíam contratos temporários, outros vínculos empregatícios, que não gozavam férias regularmente e entre aqueles que atuavam nas UTI's. Condições de trabalho inadequadas podem desenvolver insatisfação, insegurança e falta de motivação relacionada ao ambiente laboral. Além disso, ambientes de UTI's expõem o profissional a riscos físicos e químicos de maneira mais intensa do que nos outros ambientes do hospital, além de oferecer assistência a casos de maior complexidade. . $^{82}$

Foram encontradas nesta pesquisa correlações moderadas entre o número de atendimentos diários e EE, e fracas com RP. Estes resultados apresentam coerência dentro da realidade da atuação do Fisioterapeuta no âmbito hospitalar, indicando que quanto maior o número de pacientes atendidos por dia maior a EE e menor RP. Ou seja, o profissional tende a desenvolver uma autoimagem negativa relacionada ao seu desempenho no ambiente de trabalho. 8,21

Analisando-se a média de atendimentos diários $(12,83 \pm 5,45 \mathrm{DP})$ observou-se neste estudo que de maneira geral os profissionais prestam assistência a um número um pouco maior do que o preconizado pela Resolução $N^{\circ} 387$ de 08 de julho de 2011, que visa estabelecer parâmetros assistenciais nas diversas modalidades prestadas pelo fisioterapeuta. De acordo com esta resolução, - Fisioterapeuta que atua em âmbito hospitalar, a nível ambulatorial, deve atender entre 8 a 12 pacientes, de acordo com o nível de independência funcional, enquanto os que atuam em enfermarias e UTI's devem atender entre 6 e 8 pacientes a cada 6 horas. ${ }^{24}$

Apesar da média de atendimentos diários não ter sido muito superior ao preconizado pela legislação, ainda assim houve correlação com duas dimensões (EE e RP) que indicam a presença da $S B(r=-0,29, p=0,01)$. Além disso, observando-se individualmente, verificase que alguns profissionais, sobretudo os que atuavam em UTI's, chegavam a atender 
um número muito superior ao preconizado, ultrapassando 20 atendimentos por plantão de 12 horas.

Observou-se ainda correlação entre a idade e a DP, indicando que quanto maior a idade do profissional menor a perda de empatia e da sensibilidade em lidar com os pacientes. Outro estudo também encontrou associações da mesma natureza entre a DP e a idade de Fisioterapeutas que atuam em âmbito hospitalar $(r=-0,90, p=0,02) .^{21}$

Os resultados observados tornam-se relevantes uma vez que descrevem a SB em Fisioterapeutas através da utilização do $\mathrm{MBI}$ que é instrumento mais utilizado na literatura internacional para identificar a doença. Além de revelar associações entre o quadro e variáveis demográficas e laborais que podem realmente influenciar no desempenho profissional, na $E E$ e física, na sua relação com o paciente e na sua autossatisfação profissional. Além disso, este tipo de estudo envolvendo Fisioterapeutas ainda é escasso no Brasil.

Alguns Fisioterapeutas não aceitaram participar do estudo e outros não foram encontrados, porém mais de $90 \%$ dos profissionais foram incluídos. Além disso, estudos transversais não permitem estabelecer relações de causa e efeito, apenas possibilitam a observação de associações entre as variáveis analisadas. Entretanto, levantam hipóteses que podem ser verificadas em estudos posteriores que visem determinar estratégias de prevenção e tratamento específicos para a população.

Diante disto, sugere-se que em estudos futuros sejam incluídos outros serviços hospitalares, possibilitando também a comparação de setores públicos e privados. Além disso, que o tamanho da amostra seja baseado em cálculos amostrais para que seja possível determinar prevalências da SB em fisioterapeutas.

\section{CONCLUSÃO}

A SB foi verificada em mais da metade dos Fisioterapeutas participantes. Em profissionais que atuavam nas UTI's, não gozavam férias regularmente, possuíam vínculos temporários com o hospital e possuíam outros vínculos empregatícios foram observadas maiores frequências da síndrome de Burnout. Correlações positivas foram observadas entre o número de atendimentos diários e a exaustão emocional e correlação negativa entre a idade com despersonalização e número de atendimentos diários com a realização pessoal. Estas associações servem de base para a realização de estudos futuros com amostras representativas para a identificação de relações de causa e efeito e a prevalência da SB em fisioterapeutas, além de determinação de estratégias de prevenção e tratamento específicas para esta classe de profissionais.

\section{REFERÊNCIAS}

1. Gianasi LBS, Oliveira DC. A síndrome de Burnout suas representações entre profissionais de saúde. Estud Pesq Psicol. 2014;14(3):756-72.

2. Santos SMP, Sousa V, Rueda FJM. Burnout e sua relação com o clima organizacional em funcionários de um hospital. ABCS Health Sci. 2015; 40(1):11-5 DOI: https://doi.org/10.7322/abcshs.v40i1.697

3. Silva ALP, Colleta TCD, Ono HRB, Woitas LR, Soare $\mathrm{SH}$, Andrade VLA, et al. Síndrome de Burnout: consequências e implicações de uma realidade cada vez mais prevalente na vida dos profissionais de saúde. Rev Bras Med Trab. 2016;14(3):275-84. DOI https://doi.org/10.5327/Z1679-443520163215

4. Previdência Social [homepage na Internet]. Brasília: Ministério da Previdência Social; c2107 [citado 2017 Ago 05]. Disponível em: http://www.previdencia.gov. br/dados-abertos/estatsticas/tabelas-cid-10/

5. Maslach C, Jackson SE. The measurement of experienced Burnout. J Occup Behav. 1981;2(2):99 113. DOI: https://doi.org/10.1002/job.4030020205

6. Carlotto MS, Câmara SG. Análise da produção científica sobre a síndrome de Burnout no Brasil. PSICO. 2008;39(2):152-158.

7. Sánchez CAM, Claro RML, Lorenzo MC, Martín $\mathrm{CV}$, Morales MA, Fernández MJF. Prevalencia de síndrome de Burnout en fisioterapia. Fisioter 2006;28(1):17-22. DOI: https://doi.org/10.1016/ S0211-5638(06)74017-2

8. Al-Imam DM, Al-Sobayel HI. The prevalence and severity of Burnout among physiotherapists in an Arabian setting and the influence of organizational factors: an observational study. J Phys Ther Sci. 2014;26(8):1193-8. DOI: https://doi.org/10.1589/ jpts.26.1193

9. Fabichak C, Silva-Junior JS, Morrone LC. Síndrome de Burnout em médicos residentes e preditores organizacionais do trabalho. Rev Bras Med Trab. 2014;12(2):79-84.

10. Metzker CAB, Moraes LFR, Pereira LZ. O fisioterapeuta e o estresse no trabalho: estudo em um hospital filantrópico de Belo Horizonte - MG. Rev Gestão Tecnol. 2012;12(3):174-96.

11. Gil-Monte PR, Peiró JM. Perspectivas teóricas y modelos interpretativos para el estudio del síndrome de Quemarse por el trabajo. Anales Psicol. 1999;15(2):261-8.

12. Carlotto MS, Camara SG. Propriedades psicométricas do Maslach Burnout Inventory em uma amostra multifuncional. Est Psicol. 2007;24(3): 325-32.

13. Benevides-Pereira AMT. MBI - Maslach Burnout Inventory e suas adaptações para o Brasil [resumo]. In: 32a Reunião Anual de Psicologia; 2001; Rio de Janeiro. Anais. Rio de Janeiro: Sociedade Brasileira de Psicologia; 2001. p.84-5.

14. Tamayo RM. Relação entre a síndrome de Burnout e os valores organizacionais no pessoal de enfermagem de dois hospitais públicos [tese]. Brasília: Universidade de Brasília; 1997.

15. Luna JMR. Síndrome de "burn out" ¿ El médico de urgencias incansable? Rev Mexicana Med Urgencias. 2002;1(2):48-56.

16. Gómez MMN, Dodino CN, Aponte CF, Caycedo $\mathrm{CE}$, Riveros MP, Martínez MPM, et al. Relación entre perfil psicológico, calidad de vida y estrés asistencial en personal de enfermería. Univ. Psychol.2005;4(1):63-75.

17. Moreira DS, Magnago RF, Sakae TM, Magajewski FR. Prevalence of burnout syndrome in nursing staff in a large hospital in south of Brazil. Cad Saude Publica. 2009;25(7):1559-68.

18. França FM, Ferrari R, Ferrari DC, Alves ED. Burnout and labour aspects in the nursing teams at two medium-sized hospitals. Rev Lat Am Enfermagem. 2012;20(5):961-70.

19. Barroso SM, Guerra ARP. Burnout e qualidade de vida de agentes comunitários de saúde de Caetanópolis (MG). Cad Saúde Colet. 2013; 21(3):338-45.

20. Grunfeld E, Whelan TJ, Zitzelsberger L, Willan AR, Montesanto B, Evans WK. Cancer care workers in Ontario: prevalence of burnout, job stress and job satisfaction. CMAJ. 2000;163(2):166-9.

21. Pavlakis A, Raftopoulos V, Theodorou M. Burnout syndrome in Cypriot physiotherapists: a national survey. BMC Health Serv Res. 2010;10:63.

22. Silva TLA, Alchieri JC. Socioeconomic and demographic aspects related to stress and the burnout syndrome among Brazilian physiotherapists. Salud Mental. 2014;37(3):227-32.

23. Ogiwara S, Hayashi $H$. Burnout amongst physiotherapists in Ishikawa Prefecture. J Phys Ther Sci. 2002;14(1):7-13.

24. Conselho Federal de Fisioterapia e Terapia Ocupacional [homepage na Internet]. Brasília: COFFITO; c2015 [citado 2015 Nov 20] Disponível em: https://www.coffito.gov.br/ nsite/?p=3150\#more-3150 